indispensable si l'on veut éviter des pertes. La trypanosomiase bovine, diagnostiquée en 1918. a dû être introduite autrefois en Guyane par des zébus importés du Sénégal dans le but d'améliorer l'élevage local. Des recherches récentes, faites par l'Institut Pasteur, ont montré que la maladie est étendue à toute la colonie.

La piroplasmose bovine existe à l'état endémique.
D'autres maladies, déjà soupçonnées, seront très probablement identifiées par la suite, il en est ainsi notamment de la pasteurellose, du charbon symptomatique.

Ces considérations sommaires sur l'état sanitaire nous permettent de conclure que le développement de l'élevage en Guyane implique d'abord l'existence d'un service vétérinaire bien organisé en personnel et matériel.

\title{
L'élevage du Mouton à Madagascar
}

\section{I. - LA RACE OVINE DE MADAGISCAR}

TOUS les moutons de Madagascar appartiennent à la même race : Ovis dolichura, variété asiatique. Leurs réserves de graisse se forment au niveau de la queue : ce sont donc des moutons à grosse queue. Voici leur description :

Tête : profil droit ou légèrement busqué, étroite, oreilles pendantes.

Cornes : chez les mâles, mais ils en sont fréquemment dépourvus.

Encolure : longue et sèche.

Poitrine : apiatie.

Croupe : inclinée.

Gigot : maigre.

Jambes : fines et convenant à une marche rapide.

Queue : grosse et longue, la graisse s'accumule sur toute la longueur ce qui lui donne une apparence plus ou moins cylindrique. C'est surtout la partie supérieure qui est hypertrophiée dans ses deux tiers; la partie inférieure est épaisse également, mais à un degré moindre et se relève au niveau des jarrets et forme souvent une demi-spirale jusqu'à sa terminaison. La queue est couverte de poils à sa face supérieure et nue à sa face inférieure; elle est plus ou moins tendue suivant l'état d'embonpoint des animaux, d'un poids variable aussi avec la taille de ceux-ci et qui peut atteindre deux ou trois kilos, quelquefois plus. Cette graisse constitue l'aliment de réserve pour la saison sèche.

Toison : elle ressemble beaucoup à celle de la chèvre et est formée de poils plus ou moins longs, grossiers, ternes, durs, raides et qui ne presentent aucun intérêt pour le tissage.

Couleur du pelage : il est de couleur blanche ou café au lait avec souvent de larges taches rouges, brunes ou noires. La tête, le cou et les pattes sont noires. Les robes pie noir ou pie rouge avec des taches multiples à la tête et aux extrémités sont fréquentes.
Ces animaux sont sobres, rustiques et bons marcheurs. Ils s'entretiennent facilement, mais présentent de nombreux défauts : absence de laine, mauvaise conformation, viande de qualité plutôt médiocre, et également format un peu faible.

Taille et poids. $A$ ces points de vue; on peut les diviser en deux 'groupes.

$I^{\text {er }}$ groupe. - Mouton des Hauts Plateaux. - Leur poids varie de 15 à 35 kilos avec une production de viande de 9 à 20 kilos. La taille oscille entre 55 et 63 centimètres et la longueur de $0 \mathrm{~m}, 95$ à $1 \mathrm{~m}$. 05 . Le poids du cuir sec va de $1 \mathrm{~kg} .300$ à $1 \mathrm{~kg} .800$.

$2^{\mathrm{e}}$ groupe. - Moutons de l'extrême-sud (Androy et Mahafaly). - Les moutons sont plus gros et plus grands. Le poids varie de 35 à 45 kilos et la taille de $0 \mathrm{~m} .70$ à $0 \mathrm{~m} .95$.

\section{II. - RÉGIONS D'ÉLEVAGE}

Il y a deux gros centres d'élevage, la région des Hauts Plateaux (Emyrne, Betsilec et pays Bara) et la région du sud (Androy, Mahafaly et Ankazoabo). Le recensement donnait, en 1940, les résultats suivants :

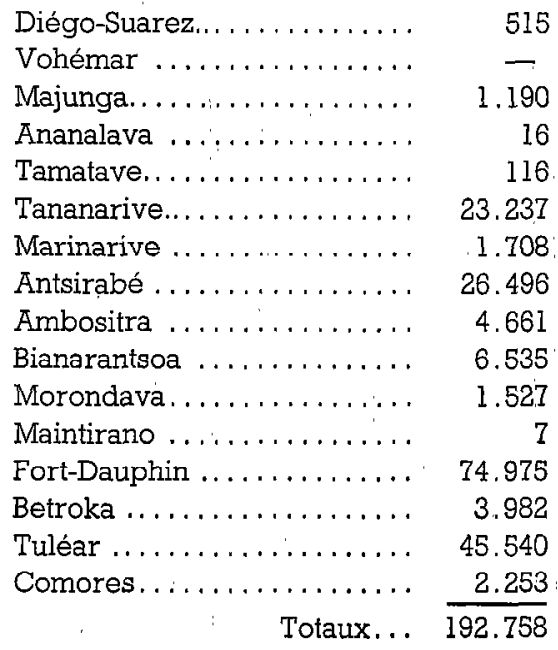


Dans le Sud, il faut distinguer le cas assez particulier de la province de Fort-Dauphin dont voici quelques chiffres successifs de recensements.

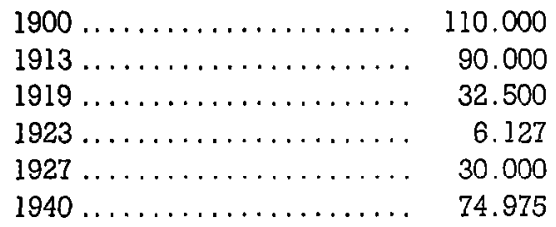

Cette province a subi en 1920 une disette qui réduisit presque à zéro son cheptel ovin. Depuis, un sérieux redressement a été opéré. La province de Tuléar compte en 1940: 45.540 moutons, contre 64.047 en 1900. Il y a donc également une assez sérieuse régression de l'élevage ovin.

Il convient de n'attacher qu'une valeur toute relative aux indications des recensements officiels. Elles servent à indiquer l'évolution du cheptel, sans toutefois donner les chiffres exacts. On peut estimer que Madagascar comptait en réalité environ 300.000 moutons.

Nous avons déjà vu que les animaux élevés dans les deux zones sont assez différents, il en est de même pour les méthodes d'élevage et pour l'alimentation. Nous étudierons donc séparément dans les chapitres suivants les conditions de l'élevage sur les Hauts Plateaux et dans la région Sud.

\section{Région da Sud}

a) Constitution géologique. - La plus grando partie des sols constituant l'extrême-sud de Madagascar sont des terrains éruptifs et métamorphiques, flanqués a l'ouest par quelques terrains de la fin du primaire (carbonifère et permien) et au sud et à l'ouest en bordure de la côte, il y a une zone de terrains alluvionnaires. Cette dernière zone, assez large au sud, est plus réduite dans l'ouest. Il convient également de signaler dans la province de FortDauphin un gros noyau de terrains éruptifs.

Au total, nous avons donc surtout des terrains granitiques et schisteux, donnant un sol assez médiocre et pauvre en calcaire.

Le relief est assez peu marqué, sauf dans la région volcanique de la province de Fort-Dauphin où il y a quelques sommets culminants vers 1.800 mètres. D'une manière générale, ce sont des plateaux d'une altitude moyenne de 100 à 200 mètres.

b) Climatologie. - Nous rencontrons principalement, dans la zone d'élevage du mouton, deux sortes de climat : le climat sud et le climat ouest.

Ces deux zones sont soumises à des vents, déjà partiellement desséchés dans leur passage au-dessus des chaines de la côte est. Ce sont des zones à climat chaud, sec et relativement sain. La zone ouest est déjà plus sèche que la zone sud.
En saison sèche, le thermomètre descend à $7^{\circ}$ ou $8^{\circ}$ la nuit; le jour, il atteint 30 à $32^{\circ}$. C'est une chaleur sèche, tempérée par le vent. L'hivernage est assez court (janvier à mars) mais la chaleur des orages est pénible.

Voici quelques chiffres de chutes de pluies :

Fort-Dauphin ........ $1.777 \mathrm{~mm}$.

Ambovombé ......... $532 \mathrm{~mm}$.

Tuléar .............. $493 \mathrm{~mm}$.

Betroka ............. $748 \mathrm{~mm}$

Tsivory ............ 1:070 mm.

Températures moyennes :

\begin{tabular}{|c|c|c|c|c|c|}
\hline \multirow{2}{*}{ STA TIONS } & \multicolumn{4}{|c|}{ MOYENNES } \\
\cline { 2 - 6 } & annuelle & de déc. & de janv. & de juill. & d'août \\
\hline Fort-Dauphin & $23^{\circ} 3$ & - & $29^{\circ} 8$ & $15^{\circ} 6$ & - \\
Tuléar ....... & 25 & - & $33^{\circ} 3$ & - & $14^{\circ} 4$ \\
Betroka ...... & $25^{\circ} 9$ & $32^{\circ} 7$ & - & $9^{\circ} 6$ & - \\
\hline
\end{tabular}

Les écarts entre l'été et l'hiver sont donc bien marqués et s'accentuent de l'est vers l'ouest et de la côte vers l'intérieur.

c) Flore agrostologique. - Dans les parties les plus humides, nous trouvons une prairie à

Heteropogon contortus (nom vulgaire : Anidambo) qui est maintenue par le bétail à l'état de prairie rase. En plus de cette graminée, on trouve également :

Poa annua;

Cymbopogon rufus;

Imperata arundinacea;

Andropogon intermedius;

Andropogon hirtus.

Dans les régions plus sèches à climat subdésertique, nous trouvons principalement :

Panicum Voeltzkowii;

Digitaria sp.;

Atriplex sp. (région de Mahafaly);

Paspalum distichum (région du lac Tsimanampetsosa):

Opuntia vulgaris;

Euphorbia stenolada:

Celastrus;

Opuntia inermis (Raiketa) qui couvre de vastes zones.

d) Facteurs humains. - Les habitants de cette région (Antandroy et Mahafaly) sont d'assez bons éleveurs et quoiqu'ils portent surtout leur attention vers l'élevage des bovins, les moutons sont en général l'objet d'une certaine surveillance. 


\section{Région des Hauts Plateaux}

a) Constitution géologique. - Nous nous trouvons également ici en présence de terrains primitifs, principalement des granits et des gneiss. Il convient toutefois de signaler un important massif volcanique dans le sud de la province de Tananarive.

Seules les vallées comportent quelques dépôts récents, mais au total de peu d'étendue.

Nous avons donc un sol pauvre et imperméable, conservant bien l'humidité et favorisant l'existence de nombreux parasites du mouton dans les mares. Évidemment, ces terrains sont très pauvres en calcaire.

Le relief est assejz accidenté. Les Hauts Plateaux dont l'altitude moyenne est de 1.400 à 1.500 mètres sont surmontés par de nombreux pics pouvant atteindre 2.000 mètres. Le sud de la province de Tananarive est particulièrement accidenté.

b) Climatologie. - Le climat de cette région est appelé climat du centre. IJ est caractérisé par une saison des pluies (novembre à avril) chaude et très orageuse, et par ưne une saison froide accompagnée de brume (erika) et de brouillards.

Voici quelques moyennes de température :

\begin{tabular}{|c|c|c|c|c|}
\hline \multicolumn{2}{|c|}{ Stations } & \multicolumn{3}{c|}{ MOYENNES } \\
\hline & annuelie & d'oct. & de nov. & de juil. \\
\hline Tananarive........ & $24^{\circ} 8$ & - & $27^{\circ}$ & $9^{\circ} 0$ \\
Antsirabé ......... & $23^{\circ} 4$ & $27^{\circ} 4$ & - & $3^{\circ} 4$ \\
\hline
\end{tabular}

Voici quelques chiffres de chutes de pluie:

Tananarive ........... $1.360 \mathrm{~mm}$.

Antsirabé............. $1.648 \mathrm{~mm}$.

Ankozobé ............ $1,854 \mathrm{~mm}$.

Ambositra............... 1,499 mm.

c) Flore agrostologique. - Le climat de cette zone correspond normalement à une végétation très dense, mais cette végétation a été détruite par le feu de brousse qui a peu à peu ruiné le sol:

Nous avons comme stade intermédiaire des savoka à Philippia sur les pentes exposées' à l'est, et des formations à fougères et à Cymbopogon cymborius sur les pentes exposées à l'ouest.

Puis à cés formations succèdent des prairies à graminées pyrophiles et xérophiles. Voici les principales espèces (nous précédons du signe - les plantes "qui fórment la prairie définitive) :

\section{Graminées :}

\# Andropogon hirtus;

Andropogon hirtifloris;

Cymbopogon rufus;
Cymbopogon sp.;

Cymbopogon Lecomtei;

Avenastrum avenoïdes:

- Trachypogon polymorphus;

Andropogon ivohihensis;

Chrysopogon montanus;

Hulalia villosa;

Arundinella stipoïdes;

- Trichoplerix stipoïdes;

- Alloteropsis semialata;

- Pennisetum triticoïdes:

- Ctenium concinnum;

- Andropogon Madagascariensis;

- Aristida multicaudis :

- Aristida similis;

- Cyperus compactus.

Autres familles (L'égumineuses) :

Desmodium sp.;

Desmodium adscendens:

Zornia diphylla;

Briosema Bajeri;

Leptodesmia congesta;

Vigna angivẹnsis.

Autour des villages, nous trouvons des prairies rases qui ne sont pas soumises aux feux de brousse. Nous y trouvons une flore assez différente.

Prairies ombragées :

Cynodon daclylon;

Stenotaphrum madage;

Paspalum scrobiculatum;

Digitaria longiflora;

Panicum luridum;

Panicum nossibense;

Paspalum distichum.

Champs en friche:

Panicum colonum;

Digitaria sanguinalis;

Setaria glauca ;

Paspalum dilatatum;

Eragrostis abyssinica;

Tricholœna rosea;

Agrostis emirnensis.

Dans les ravins et au bord de l'eau, se réfugient les plantes suivantes :

Poa :

Festuca ;

Brachypodium;

Leptodesmia congesta;

Melinis multiflora;

Desmodium adscendens.

d) Facteurs humains. - Les indigènes des Hauts Plateaux sont d'une manière générale de bons éleveurs, mais ils se désintéressent à peu près totalement de l'élevage du mouton. 


\section{III. - CONDUTHE DE L'ÉLEVAGE \\ 10 Région da Sud}

Aucun indigène ne se consacre uniquement à I'élevage dans cette région. Chacun d'entre eux possède un troupeau de 30 à 40 têtes, qui suit les bœufs lorsque ceux-ci vont au pâturage. Le soir, les animaux sont parqués avec les breufs dans des enclos. La douceur du cumat et la faible pluviosité ne rendent pas nécessaire la construction de bergeries. La nuit, les animaux sont rentrés dans des parcs entourés de murs en troncs d'arbre oü de cactus, cela principalement pour éviter les vols.

La reproduction n'est l'objet d'aucun soin et les mâles défectueusement conformés ne sont pas castrés. Chaque brebis donne ordinairement deux agneaux, qu'ellé arrive à nourrir facilement, étant bonne laitière.

Il n'existe dans cette zone aucune bête de proie, et les infections parasitaires sont assez rares.

\section{Région des Hants Plateanx}

L'élevage n'y est pratiqué que par de très petits propriétaires; c'est un élevage familial, car les gros propriétaires préfèrent pratiquer l'élevage des boeufs, non pas parce qu'i] est plus rémunérateur, mais parce qu'il est plus honorifique.

L'élevage est pratiqué de la manière la plus simple. Les animaux broutent durant toute l'année à proximité des villages et sans gardien. Ils pâturent sur le bord des rizieres et dans celles-ci quand la moisson est faite. Ils vont également dans les marais où ils ont de l'eau jusqu'au ventre.

Leur alimentation est assez difficile pendant la saison sèche, mais il est rare qu'on leur donne alors un supplément de nourriture (son de riz, manioc; etc.).

Béliers et brebis vivent en commun durant toute l'année. Il n'y a donc aucun travail de sélection. Les naissances se font en toute saison, avec en moycnne un agneau par brebis et assez souvent deux.

La nuit, les moutons rentrent avec les boufs dans des parcs entourés de rinurs de pisé. Ces parcs, quí ont pour but d"empêcher les vols et de donner une production de fumier, ne sont jamais nettoyés et sont de véritables bourbiers.

L'élevage est donc pratiqué de la façon la plus élémentaire, et il est compréhensible que dans ces conditions les animaux, non seulement ne soient pas d'une qualité remarquable, mais en plus soient fréquemment atteints de parasites ou attaqués par des épizooties.

\section{IV. - PAThologie}

Les conditions climatiques établissent une nette différence entre l'état sanitaire des troupeaux du
Sud et celui des troupeaux des Hauts Plateaux. Nous les étudierons donc séparément.

\section{Région du Sud}

Les affections pathologiques atteignent très peu le mouton dans cette région.

\section{a) Maladies parasitaires:}

- La strongylose de la caillette, qui est la maladie la plus fréquente, fait de temps en temps des dégâts, chez les agneaux, lors d'années: anormalement pluvieuses.

- L'œsophagostomose est fréquente, mais ne semble guère gêner les animaux.

- La douve est très rare.

- L'œstrose des sinus est très fréquente et amaigrit les animaux, à la saison où les larves ayant atteint leur plein développement sont une grosse gêne pour la respiration.

- La gale psoroptique s'observe pendant les années pluvieuses.

\section{b) Maladies infectieuses :}

- Le charbon est rare dans toute cette région.

- La Heart-Water est à peu près inconnue.

- Une conjonctivite enzootique, peut-être contagieuse, apparaît tous les ans au printemps.

- Le Preiz-Nocard fait de temps en temps quelques victimes.

En résumé, seules les maladies parasitaires sont à craindre pendant les années exceptionnellement pluvieuses.

Il faut ajouter les rares accidents imputables à des végétaux. Les cactus épineux, dont les longs piquants peuvent provoquer des abcès en pénétrant dans la peau ou sous les sabots, sont presque totalement disparus.

Une graminée, l'Ahidambo; a des épillets qui pénètrent dans la toison et peuvent progresser jusqu'au contact de la peau qu'ils irritent et ils peuvent être la cause d'abcès. Heureuscmont, ils n'atteignent leur maturité que peu de temps avant la tonte, et les pasteurs indigènes savent éviter les champs où poussent ces graminées.

\section{$2^{\circ}$ Région des Hauts Plateaux}

L'humidité et le sol imperméable de cette région expliquent l'importance considérable des maladies parasitaires qui peuvent atteindre gravement le troupeau.

\section{a) Maladies parasitaires :}

- La Heart-Water, cause des pertes nombreuses dans le troupeau, surtout chez les animaux importés.

- La strongylose, l'œsophagostomose, l'œstrose 
et la gale psoroptique sont relativement beaucoup plus fréquentes que dans la zone sud.

Il convient également de signaler :

- La douve du foie, assez répandue.

b) Maladies infectieuses :

- Le charbon bactéridien est plutôt rare et n'atteint guère que les animaux importés.

- La lymphangite ulcéreuse à Preiz-Nocard, sévit avec une certaine intensité et cause d'assez nombreuses morts.

On ne signale comme plante nuisible que Sporobolus indicus ou toiana:

Nous voyons donc que d'une manière générale la zone des Hauts Plateaux est beaucoup moins saine que la zone sud. Ceci est particulièrement marqué chez les animaux importés, car les moutons indigènes ont une certaine immunité naturelle.

\section{V. - LES ESSAIS D'AMÉLIORATION DU CHEPTEL OVIN}

Dès le début de l'occupation française à Madagascar, des essais ont été effectués pour améliorer les moutons indigènes et en particulier pour essayer d'obtenir des moutons à laine. Nous ne rappellerons pas en détail ces travaux, et nous nous contenterons de présenter le bilan tel qu'il pouvait être dressé en 1940 pour chacune des deux zones à moutons.

\section{$1{ }^{\circ}$ Région du Sud}

Avant la guerre de 1914, quelques essais d'introduction de mérinos de Provence, de South down et de mérinos de Rambouillet échouaient, en partie par manque de persévérance.

La chambre de commerce de Tourcoing décidait après la guerre de tenter de créer un élevage de moutons à laine de race pure. Elle choisit pour cela le mérinos d'Afrique du Sud et l'exploitation fut fixée à Andriandampy, sur le plateau de l'Horombe, au nord de Betroka. Les animaux ainsi introduits durent vivre avec le seul pâturage naturel comme alimentation. Aussi, très rapidement, furent-ils victimes d'épidémies de Heart-Water, de strongylose de la caillette, d'œesophagostomose, etc.

Cet élevage dut être interrompu en 1928

En 1921, le Service de l'Élevage avait créé une bergerio officielle à Ambovombc (province de Fort-Dauphin). Les expériences qui y furent entreprises furent menées selon un principe différent. On chercha à améliorer le cheptel indigène par croisement avec des moutons à laine. On introduisit pour cela des mérinos du Châtillonnais et des mérinos de Provence.

Les Châtillonnais résistèrent très mal et cet essai fut abandonné. Mais avec les mérinos de Provence les résultats furent plus intéressants et les métis obtenus semblent devoir donner satisfaction.

Le nombre des mérinos de race pure est passé de 32 en 1924 à 292 en 1937, et celui des métis, de 324 en 1927 à 4.972 en 1937.

Le pourcentage des naissances par rapport aux femelles a atteint $96 \%$ en 1937, à la station d'Ambovombe.

Ces essais montrent qu'en prenant les précautions nécessaires contre les parasites et en donnant aux animaux une alimentation régulière, l'élevage du mouton à laine est possible dans la région Sud.

\section{Région des Hauts Plateaux}

Là aussi, de nombreux essais d'acclimatation du mouton à laine ont été entrepris, mais ils se sont heurtés surtout à des difficultés provenant de l'abondance des parasites et des maladies parasitaires.

Toutefois, la ferme de Faratsiho (Province de Tananarive) a entrepris le croisement avec des moutons de race Dishley-Mérinos (mérinos d'Ile-deFrance), pour avoir des produits donnant à la fois de la laine et une viande de bonne qualité.

Les résultats ne sont pas aussi interessants que ceux obtenus dans le Sud. La natalité ne s'est élevée, en 1937, qu'à $60 \%$. Les produits restent assez sensibles aux parasites. Il semble que l'avenir soit plutôt dans la production d'animaux à viande, améliorés par croisement industriel. 